\title{
A novel vascular endothelial growth factor-trap KP-VR2 with enhanced ligand blocking
}

\author{
Mi-Ra Kim ${ }^{1,2}$, Bongkyu kim ${ }^{1,2}$, Yunsu Na ${ }^{1}$, Jingon $\mathrm{Yoo}^{1}$, Se-Ho Kim², In-Ra Seo ${ }^{1 *}$
}

${ }^{1}$ Biotechnology Research Division, Korea Prime Pharm. Co., LTD, Suwon 16229, Republic of Korea

${ }^{2}$ Division of Biomedical Convergence, College of Biomedical Science, Kangwon National University, Chuncheon 24341, Republic of Korea

*Corresponding Author: In-Ra Seo;

E-mail: ir.seo@koreaprime.co.kr

Running Title: Vascular endothelial growth factor-trap KP-VR2

Keywords: Angiogenesis; KP-VR2; Placental growth factor; Vascular endothelial growth factor; Vascular endothelial growth factor receptor; VEGF-Trap 


\begin{abstract}
Antiangiogenic therapies targeting vascular endothelial growth factor (VEGF)-A have been commonly used in clinics to treat cancers over the past decade. However, their clinical efficacy has been limited, with drawbacks including the acquisition of resistance and activation of compensatory pathways resulting from elevated circulating VEGF-B and placental growth factor (PlGF) levels. Thus, we developed a novel VEGF-Trap, KP-VR2, which can neutralize VEGF-A, VEGF-B, and PIGF to mediate these problems. KP-VR2 consists of two consecutive second Ig-like domains (D2s) of VEGF receptor 1 (VEGFR-1) fused to human IgG1 Fc. KP-VR2 showed more potent decoy activity than the current VEGF-Trap against VEGF and PIGF. Most importantly, two consecutive D2s of VEGFR-1 can generate two putative binding sites, resulting in a significant improvement in binding capacity. These advances resulted in stronger antitumor efficacy in implanted tumor models than aflibercept and bevacizumab. Overall, the results of this study highlight KP-VR2 as a promising therapeutic candidate for further clinical drug development.
\end{abstract}


bioRxiv preprint doi: https://doi.org/10.1101/2021.02.02.429416; this version posted February 2, 2021. The copyright holder for this preprint (which was not certified by peer review) is the author/funder. All rights reserved. No reuse allowed without permission.

\section{Introduction}

Angiogenesis is defined as the neoformation of blood vessels through a multistep mechanism that provides nutrients and oxygen to tissues, allowing the discharge of waste products [1]. This process works in physiological conditions, such as wound healing, embryogenesis, and inflammation, but is also crucial for pathological conditions like cancer [2]. The vascular endothelial growth factor (VEGF) is considered the most important growth factor and is a well-characterized contributor to angiogenesis [3].

VEGF represents the most important and widely studied proangiogenic factor family, and it is composed of five growth factors named VEGF-A, VEGF-B, VEGF-C, VEGF-D, and placental growth factor (PIGF) [4-6]. VEGFs play their role in angiogenesis by binding to three different receptors located on the cell membrane: VEGFR-1 (Flt-1), VEGFR-2 (Flk/KDR), and VEGFR-3 (Flt-4) [7]. VEGF-A is the most important regulator in human physiological and pathological angiogenesis, and it is related to a poor prognosis in several cancers [8]. VEGF-A may interact with both VEGFR-1 and VEGFR-2, but due to the limited kinase activity of VEGFR-1, the active kinase activity of VEGFR-2 makes it the most important effector of VEGF-A downstream signaling [9]. VEGF-B, which has common structural homology with VEGF-A and whose activity is mediated by interaction with VEGFR-1, plays a role in tumorigenesis and blood vessel survival under stress conditions [10]. VEGF$\mathrm{C}$ and VEGF-D, which bind VEGFR-3, are involved in lymphangiogenesis [11].

PlGF is another important growth factor that directly regulates vessel growth and maturation by affecting endothelial cells and indirectly regulates these by recruiting proangiogenic cell types. PlGF shares structural homology with VEGF-A and stimulates angiogenesis through interaction with VEGFR-1 [12-15]. Unlike VEGF-A, which binds to both VEGFR-1 and VEGFR-2, PlGF binds to VEGFR-1 but not to VEGFR-2 [7].

Aflibercept, also known as ziv-aflibercept or VEGF-Trap (Eylea ${ }^{\circledR}$ and Zaltrap ${ }^{\circledR}$, Regeneron Pharmaceuticals, Tarrytown, NY, USA and Sanofi-Aventis, Bridgewater, NJ, USA, respectively), has been approved by the US Food and Drug Administration (FDA) for the treatment of macular degeneration and metastatic colorectal cancer [16]. Aflibercept is a recombinant fusion protein consisting of the second immunoglobulin (Ig) domain of VEGFR-1 and the third Ig domain of VEGFR2 fused to human IgG1 Fc. It exhibits affinity for VEGF-A, VEGF-B, and PlGF [3, 17].

The VEGF-Trap bevacizumab (Genentech, Inc., San Francisco, CA, USA) is a VEGF blocking monoclonal antibody and has two ligand binding sites. However, bevacizumab binds VEGF-A but not VEGF-B or PlGF.

To address the limitations of the current VEGF-Trap, we designed a new structure of VEGF-Trap KPVR2 to consist of two consecutive D2s of VEGFR-1 fused to the Fc region of human IgG1. Considering the main role of VEGFR-1 D2 and the minor role of VEGFR-2 D3 in the binding of VEGF [18], we generated KP-VR2 by replacing VEGFR-2 D3 with VEGFR-1 D2 in aflibercept. Thus, two consecutive D2s of VEGFR-1 create two putative ligand binding sites, resulting in improved decoy efficiency compared to aflibercept with one binding site. In addition, KP-VR2 exhibits a high affinity for PIGF, to which bevacizumab cannot bind. This improvement was demonstrated by binding enzyme-linked immunosorbent assay (ELISA), human umbilical vein endothelial cells (HUVECs) migration inhibition, and tumor xenograft models.

\section{Methods}

\section{Results}

Construction and Expression of KP-VR2 
We designed a novel VEGF decoy receptor fusion protein that consists of the two consecutive D2s of VEGFR-1 to create the putative two binding sites for ligands, resulting in greatly improved decoy efficiency (Fig. 1A). The KP-VR2 was produced from CHO cells and purified by protein A affinity chromatography with two additional steps of ion exchange chromatography. The purified KP-VR2 showed $\sim 120 \mathrm{kDa}$ band in a non-reduced SDS-PAGE (Fig. 1B). In western blotting, using an antibody specific to Fc (Fig. 1C), specific bands could be observed for each specified protein at $115 \mathrm{kDa}$ (lane 1, aflibercept Eylea $\left.{ }^{\circledR}\right), 115 \mathrm{kDa}$ (lane 2, aflibercept Zaltrap ${ }^{\circledR}$ ), and $120 \mathrm{kDa}$ (lane 3, KP-VR2).

\section{Figure 1A}
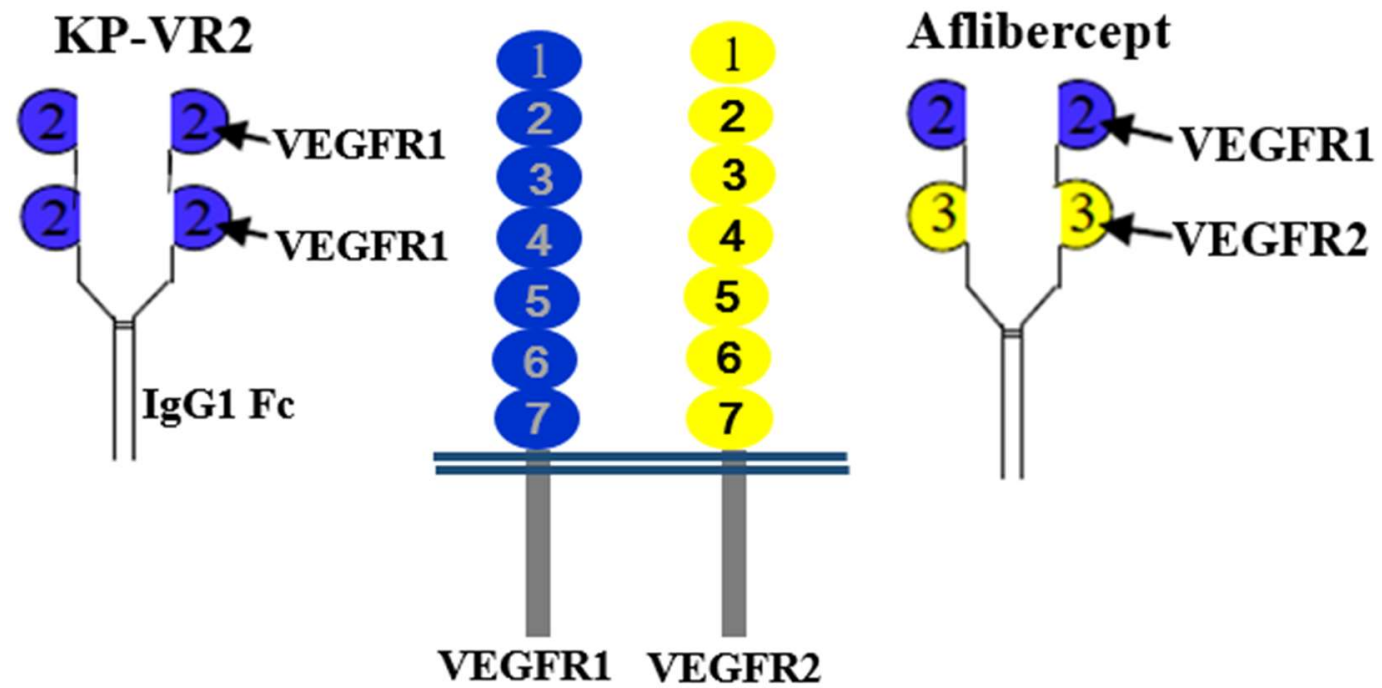

Figure 1B 


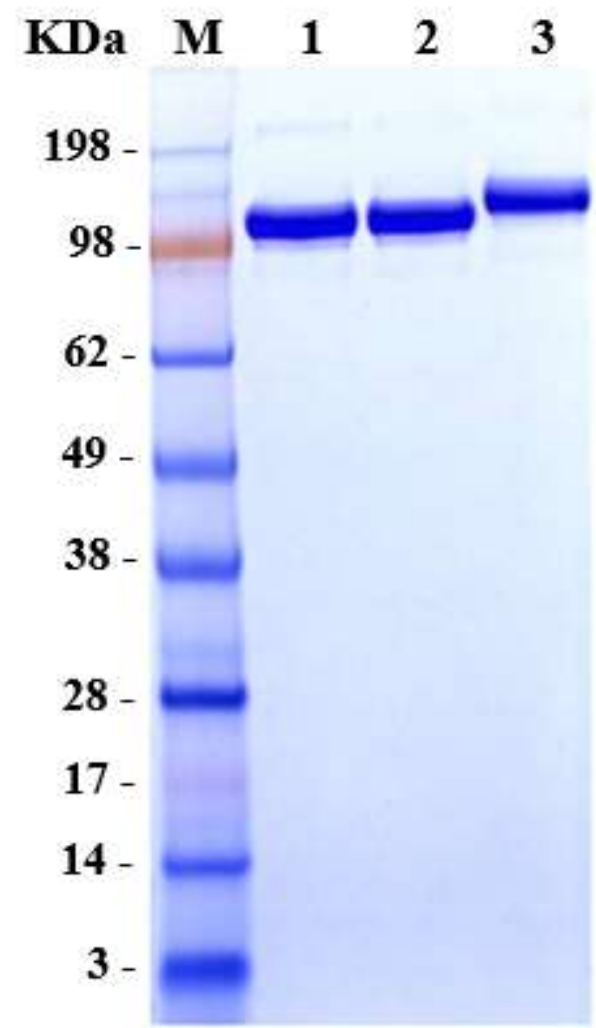

Figure 1C

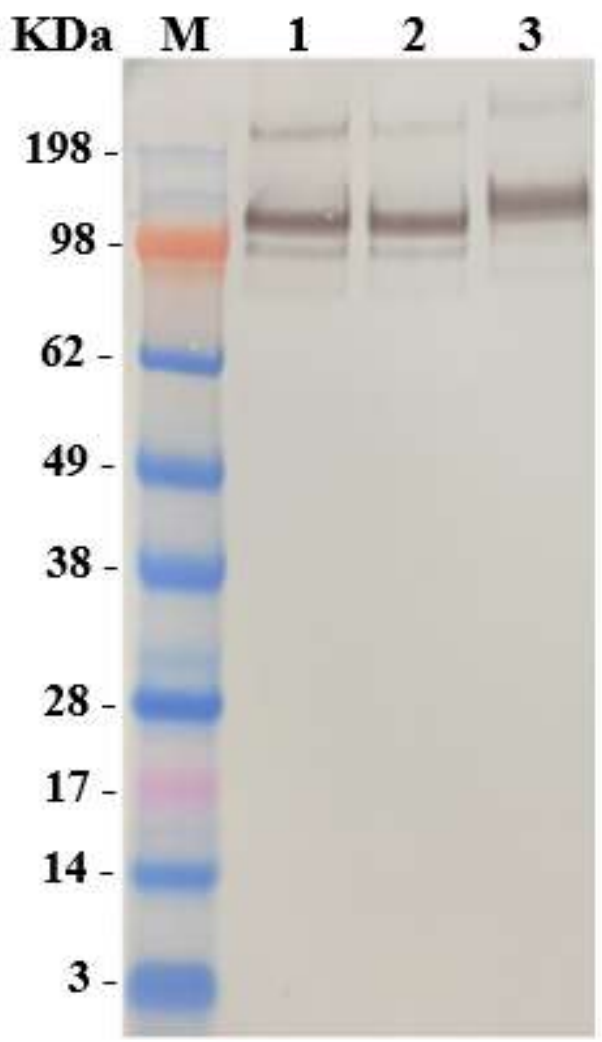




\section{Figure 1. Construction and expression of KP-VR2}

Schematic diagram of KP-VR2 and aflibercept. KP-VR2 is composed of VEGFR1 D2-D2-Fc fusion (A). SDS-PAGE analysis of aflibercept and KP-VR2 in non-reduced conditions (B). Western blot analysis of aflibercept and KP-VR2 in non-reduced conditions (C). Lanes 1, 2, and 3 represent aflibercept Eylea ${ }^{\circledR}$, aflibercept Zaltrap ${ }^{\circledR}$, and KP-VR2, respectively. Western blot analysis of KP-VR2 and aflibercept was resolved on 4-12\% gel under non-reducing conditions, transferred to a polyvinylidene difluoride membrane, and probed with $\mathrm{Fc}-$ specific antibody. For comparison, protein MW size marker is shown.

\section{Comparison of the Binding Ability of KP-VR2, Aflibercept, and Bevacizumab}

We compared KP-VR2 to aflibercept and bevacizumab to analyze its ability to bind their ligands in vitro. To determine the binding of KP-VR2, aflibercept, and bevacizumab for their ligands, such as VEGF-A 165, VEGF-A 121 , and PLGF-1, sandwich ELISA assays were performed in which an increasing amount of VEGF-A 165 and VEGF-A ${ }_{121}$ (from 0.0625 to $256 \mathrm{nM}$ ), and PLGF-1 (from 0.122 to $2000 \mathrm{nM}$ ) were reacted to immobilized KP-VR2, aflibercept, and bevacizumab. The binding was measured through incubation with Goat anti-Human VEGF antibody and then with peroxidase-conjugated antiGoat Ig antibody.

The concentration $(\mathrm{nM})$ of $50 \%$ of the maximum binding $\left(\mathrm{Bmax}_{50}\right)$ of KP-VR2, aflibercept, and bevacizumab for VEGF-A 165 was $1.66 \mathrm{nM}, 1.69 \mathrm{nM}$, and $5.16 \mathrm{nM}$, respectively (Fig. 2A and Table 1). The maximum binding optical density (Bmax O.D.) of KP-VR2, aflibercept, and bevacizumab for VEGF$\mathrm{A}_{165}$ was O.D. 1.50, 0.89, and 0.91, respectively (Fig. 2A and Table 1).

Figure 2

Figure 2A 


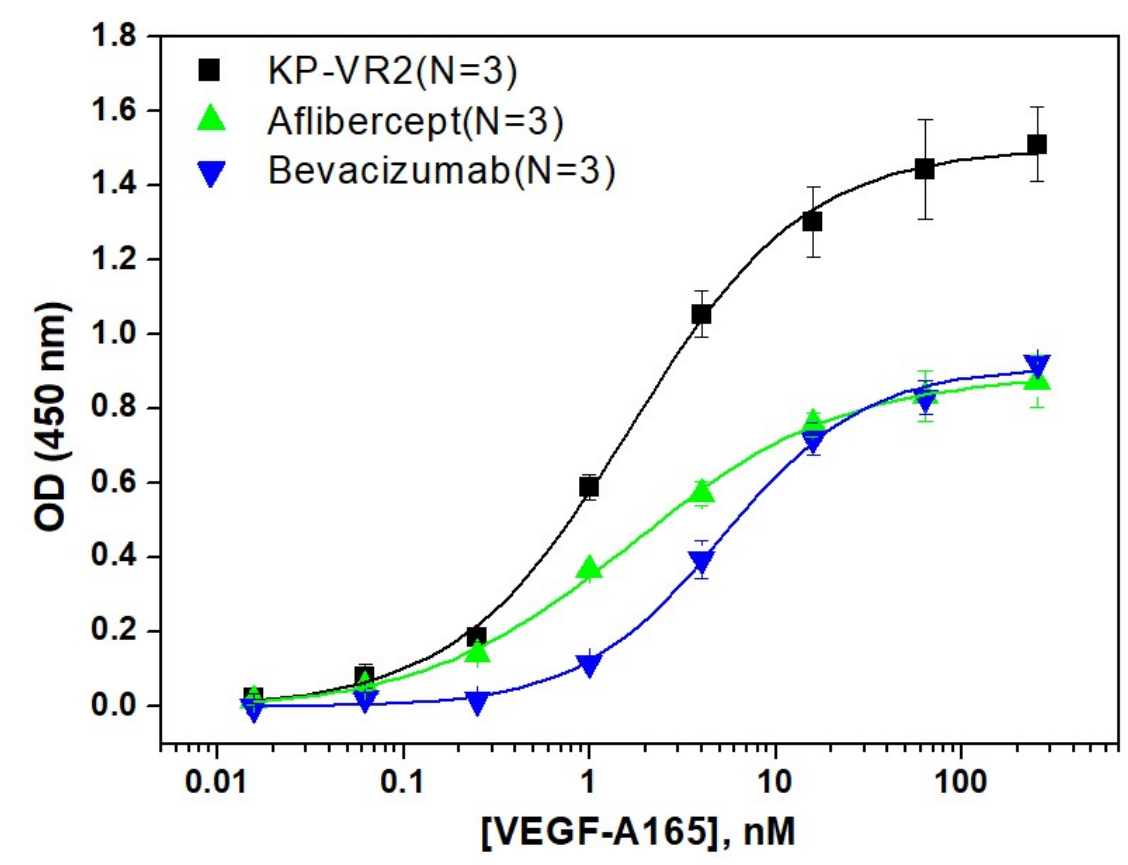

Figure 2B

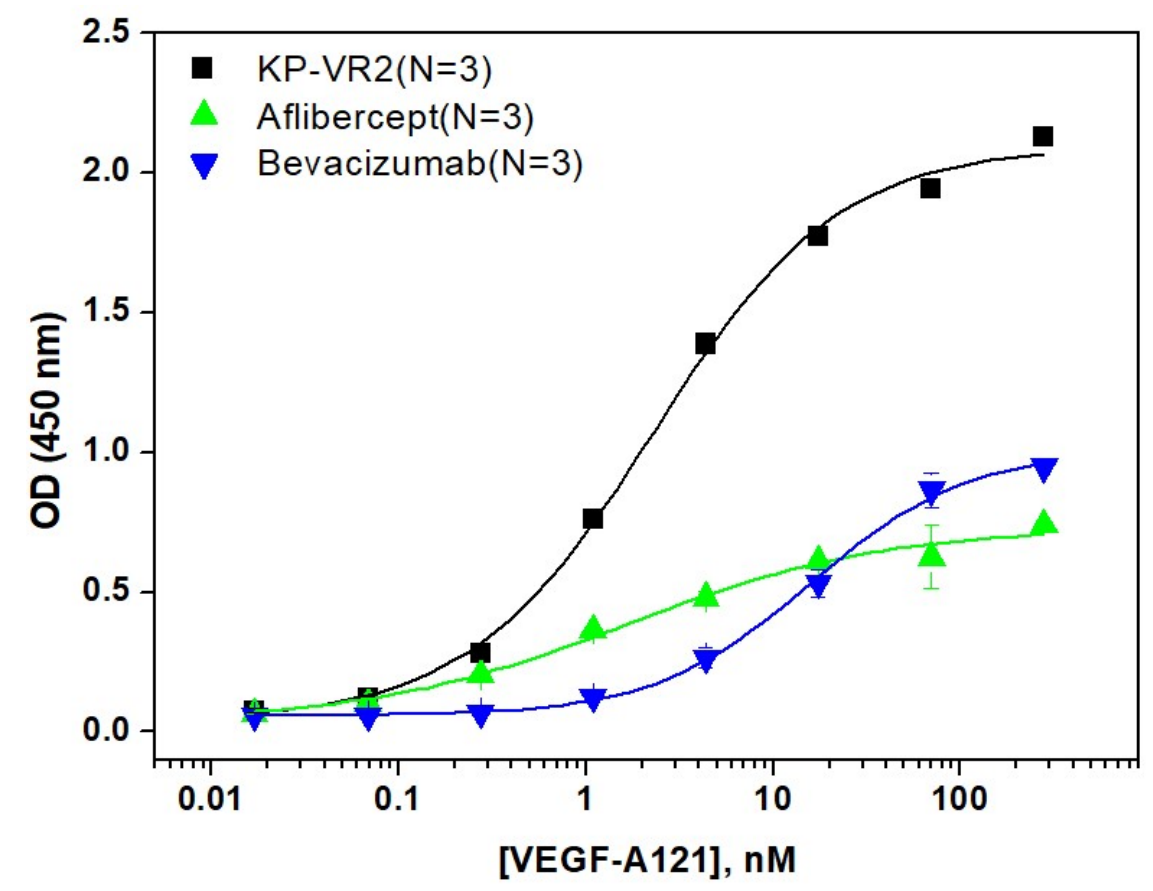

Figure 2C 


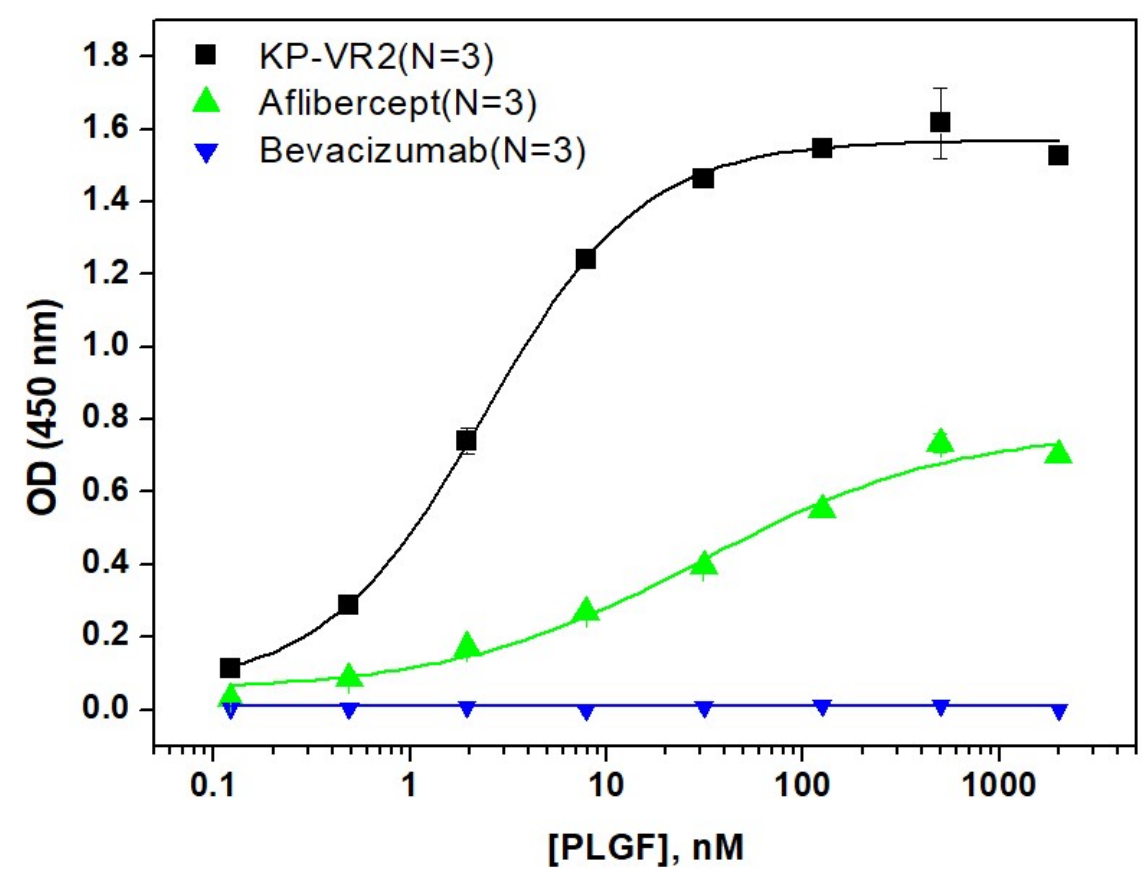

Figure 2. Analysis of binding of KP-VR2, aflibercept, and bevacizumab

Binding abilities of KP-VR2, aflibercept, and bevacizumab to VEGF-A $\mathrm{A}_{165}$ (A), VEGF-A $\mathrm{A}_{121}$ (B), and PlGF-1 (C) are presented. An increasing amount of VEGF-A $A_{165}$ and VEGF-A 1211 (from 0.0625 to $256 \mathrm{nM}$ ), and PLGF-1 (from 0.1 to $2000 \mathrm{nM}$ ) were reacted to immobilized KP-VR2, aflibercept, and bevacizumab. The binding was measured by Goat anti-Human VEGF antibody and then with peroxidase-conjugated anti-Goat Ig antibody. For each group, $n=3$. Values are presented as mean $\pm \mathrm{SD}$.

Table 1. Binding of KP-VR2, aflibercept, and bevacizumab to human VEGF family ligands by ELISA 


\begin{tabular}{lccc}
\hline KP-VR2 & VEGF-A $_{165}$ & $1.50 \pm 0.03$ & $1.66 \pm 0.15$ \\
Aflibercept & VEGF-A $_{165}$ & $0.89 \pm 0.02$ & $1.69 \pm 0.15$ \\
Bevacizumab & VEGF-A $_{165}$ & $0.91 \pm 0.02$ & $5.16 \pm 0.49$ \\
& & & \\
KP-VR2 & VEGF-A $_{121}$ & $2.10 \pm 0.04$ & $2.27 \pm 0.24$ \\
Aflibercept & VEGF-A $_{121}$ & $0.73 \pm 0.04$ & $1.63 \pm 0.50$ \\
Bevacizumab & VEGF-A & $1.01 \pm 0.03$ & $16.03 \pm 1.59$ \\
& & & \\
KP-VR2 & PLGF-1 & $1.57 \pm 0.02$ & $2.39 \pm 0.18$ \\
Aflibercept & PLGF-1 & $0.77 \pm 0.06$ & $30.47 \pm 11.46$ \\
Bevacizumab & PLGF-1 & - & -
\end{tabular}

$\mathrm{Bmax}_{\mathrm{OD}}$ and $\mathrm{Bmax}_{50}$ represent the maximum binding O.D. and the concentration of ligands at $50 \%$ of the maximum binding, respectively

The Bmax $_{50}$ of KP-VR2, aflibercept, and bevacizumab for VEGF-A ${ }_{121}$ was $2.27 \mathrm{nM}, 1.63 \mathrm{nM}$, and $16.03 \mathrm{nM}$, respectively (Fig. 2B and Table 1). The Bmax ${ }_{\text {O.D }}$ of KP-VR2, aflibercept, and bevacizumab for VEGF-A ${ }_{121}$ was O.D 2.10, 0.73, and 1.01, respectively (Fig. 2B and Table 1).

The Bmax 50 of KP-VR2 and aflibercept for PlGF-1 was $2.39 \mathrm{nM}$ and $30.47 \mathrm{nM}$, respectively (Fig. 2C and Table 1). The Bmaxo.D of KP-VR2 and aflibercept for PIGF-1 was O.D 1.57 and 0.77, respectively (Fig. 2C and Table 1). No binding and bevacizumab binding was detected for PlGF-1 and bevacizumab.

\section{Comparison of Affinity of KP-VR2 and Aflibercept for VEGF-A $A_{165}$ and PLGF-1 by Octet}

BLI analysis was performed to assess the binding kinetics of KP-VR2 to VEGF-A and PlGF using a ForteBio Octet System. KP-VR2 and aflibercept were immobilized onto biosensors, which were subsequently dipped into a dilution series of VEGF-A $\mathrm{A}_{165}$ and PlGF-1. The dynamic reactive process between analytes and ligands was automatically recorded by the Octet control software. The curve and results were analyzed according to the 1:1 binding model (aflibercept) and the 1:2 binding model (KPVR2). The dynamic association and dissociation curves are shown in Figure 3A to 3B. The kinetics and affinity constants between analytes and ligands are listed in Table 2. Notably, the equilibrium dissociation constant $\left(K_{D}\right)$ of KP-VR2 for VEGF-A $\mathrm{A}_{165}(<1 \mathrm{pM}$ and $4.75 \mathrm{pM})$ was similar to that of aflibercept (2.69 pM) (Table 2). KP-VR2 and aflibercept also bound human PlGF-1 with a $K_{D}$ of 0.78 $\mathrm{nM}$ and $25.1 \mathrm{nM}$, respectively. Aflibercept showed a binding affinity to PIGF (1.30 nM).

\section{Figure 3A}




\section{Aflibercept}

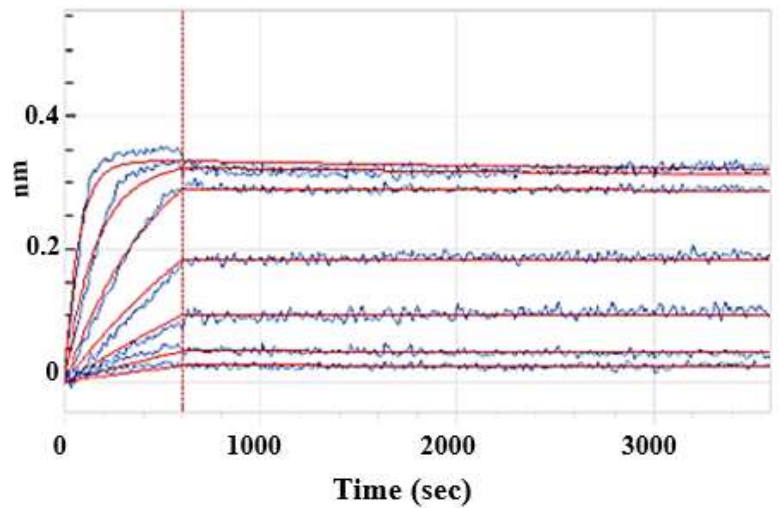

Figure 3B

\section{Aflibercept}

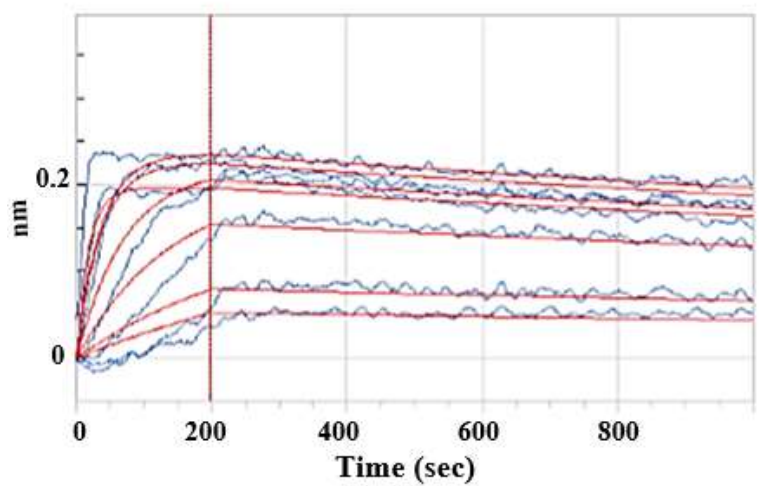

\section{KP-VR2}

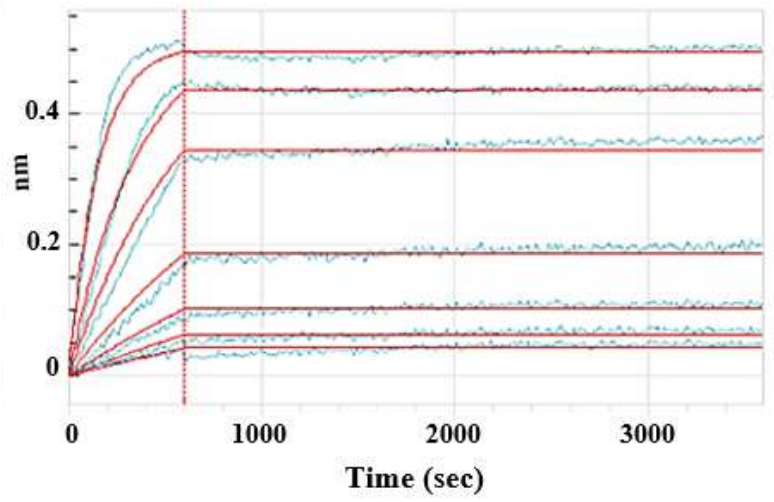

KP-VR2

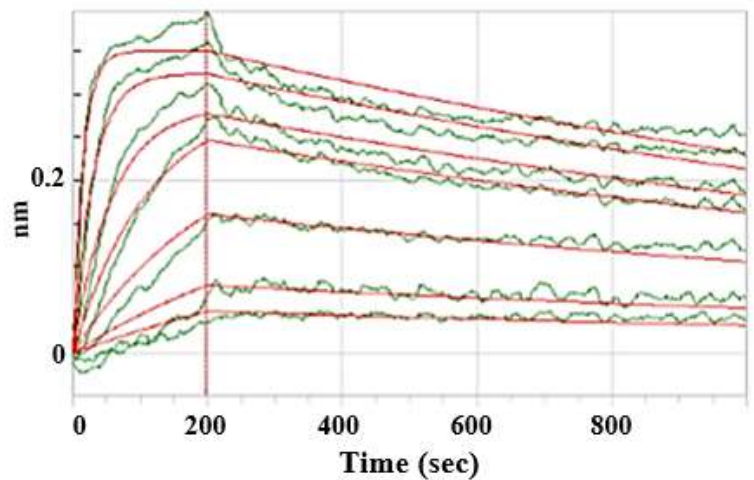

Figure 3. Binding affinities of KP-VR2 and aflibercept for human VEGF-A165 and PIGF-1

The interaction between KP-VR and aflibercept with human VEGF-A ${ }_{165}$ and PlGF-1 was measured using Octet. KP-VR2 and aflibercept bound human VEGF-A $\mathrm{A}_{165}$ with high affinity $\left(K_{\mathrm{D}}=<1 \mathrm{pM}\right.$ and $4.75 \mathrm{pM} v \mathrm{~s}$ $2.69 \mathrm{pM})(\mathrm{A}) . \mathrm{KP}-\mathrm{VR} 2$ and aflibercept also bound human P1GF-1 with high affinity $\left(K_{\mathrm{D}}=0.78 \mathrm{nM}\right.$ and $\left.25.1 \mathrm{nM}\right)$ (B). 
Table 2. Kinetic binding parameters of KP-VR2 and aflibercept to human VEGF family ligands by Octet

\begin{tabular}{ccccc}
\hline \multirow{2}{*}{ VEGF inhibitor } & \multicolumn{3}{c}{ Kigand } & \multicolumn{2}{c}{} \\
\cline { 3 - 5 } & & $\mathbf{K}_{\mathbf{o n}}(\mathbf{1} / \mathbf{m s})$ & $\mathbf{K}_{\mathbf{d i s}}(\mathbf{1} / \mathbf{s})$ & $\mathbf{K}_{\mathbf{D}}(\mathbf{M})$ \\
\hline${ }^{1}$ KP-VR2 & VEGF-A $_{165}$ & $3.35 \times 10^{4}$ & $<1.00 \times 10^{-7}$ & $<1.00 \times 10^{-12}$ \\
${ }^{2}$ KP-VR2 & VEGF-A $_{165}$ & $6.44 \times 10^{4}$ & $3.30 \times 10^{-7}$ & $4.75 \times 10^{-12}$ \\
Aflibercept & VEGF-A $_{165}$ & $6.01 \times 10^{5}$ & $1.64 \times 10^{-6}$ & $2.69 \times 10^{-12}$ \\
& & & & \\
${ }^{1}$ KP-VR2 & PLGF-1 & $3.03 \times 10^{5}$ & $2.36 \times 10^{-4}$ & $7.80 \times 10^{-10}$ \\
${ }^{2}$ KP-VR2 & PLGF-1 & $3.44 \times 10^{5}$ & $8.65 \times 10^{-3}$ & $2.51 \times 10^{-8}$ \\
Aflibercept & PLGF-1 & $1.74 \times 10^{5}$ & $2.25 \times 10^{-4}$ & $1.30 \times 10^{-9}$
\end{tabular}

Numbers represent each binding site.

VEGF inhibitor captured on a Protein A-coupled sensor chip

\section{Comparison of KP-VR2, Aflibercept, and Bevacizumab in migration inhibition}

We determined whether KP-VR2 binding to VEGF could effectively block the ability of VEGF-induced endothelial cell migration. The inhibitory effect of KP-VR2 was evaluated by endothelial cell invasion and migration assay. KP-VR2, aflibercept, and bevacizumab could completely block VEGF-A $165-$ induced HUVEC migration at the concentration of $100 \mathrm{ng} / \mathrm{mL}$. KP-VR2 had a lower half maximal inhibitory concentration $\left(\mathrm{IC}_{50}\right)$ value than aflibercept and bevacizumab (Figure $4 \mathrm{~B}$ and Table 3 ) owing to a higher inhibition potency. VEGF induced cell invasion in HUVEC, whereas KP-VR2, aflibercept, and bevacizumab inhibited VEGF-induced cell invasion (Fig. 4A). The blue-colored spot indicates VEGF-induced cell migration (Fig. 4A). KP-VR2 showed higher inhibitory activity than aflibercept and bevacizumab at the same concentration (Fig. 4B). The $\mathrm{IC}_{50}$ values of KP-VR2, aflibercept, and bevacizumab were $11 \mathrm{nM}, 20 \mathrm{nM}$, and $21 \mathrm{nM}$, respectively (Table 3), which reveals that the KP-VR2 has outstanding inhibitory activity against cell invasion.

\section{Figure 4A}




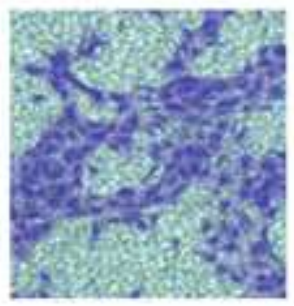

Negative

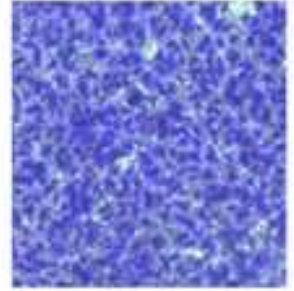

Positive

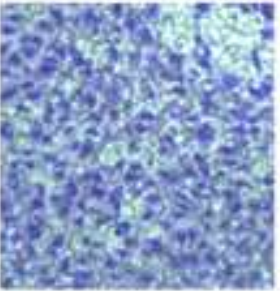

KP-VR2

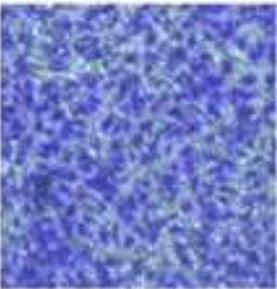

Aflibercept

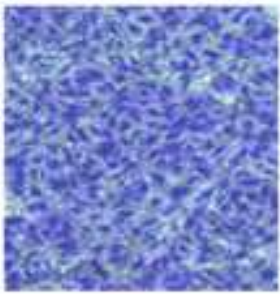

Bevacizumab

Figure 4B

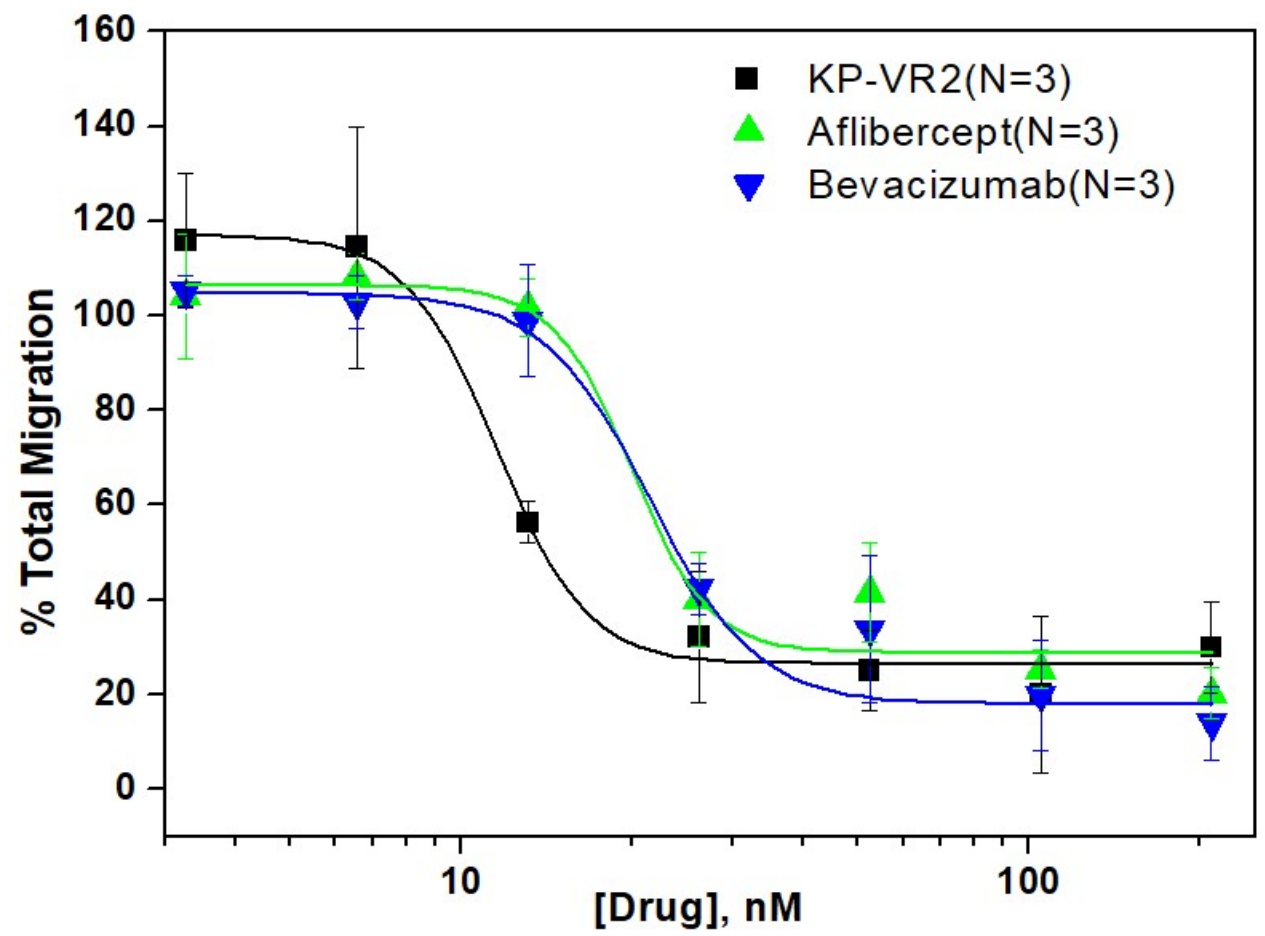

Figure 4. Inhibition of HUVEC migration by KP-VR2, aflibercept, and bevacizumab

Cell invasion assay with HUVEC in the presence of VEGF-A 165 and VEGF-Traps is presented by image (A) and quantification (B). HUVECs were placed in the upper compartment of the Boyden chamber and allowed to migrate toward basal media containing $0.1 \%$ fetal bovine serum with or without VEGF-A 165 and VEGF-A 165 mixed with four different concentrations of KP-VR2, aflibercept, and bevacizumab ranging from 3.27 to 209.44 nM. The 
percentage of total migration (y-axis) was calculated as described in the Experimental Procedures section.

Table 3. IC50 of KP-VR2, aflibercept, and bevacizumab in HUVEC migration

\begin{tabular}{llc}
\hline & Ligand & HUVEC invasion \\
\cline { 3 - 3 } & & IC $_{\mathbf{5 0}}(\mathbf{n M} \mathbf{M})$ \\
\hline & & \\
KP-VR2 & VEGF-A $_{165}$ & $11.55 \pm 0.96$ \\
Aflibercept & VEGF-A $_{165}$ & $19.93 \pm 2.62$ \\
Bevacizumab & VEGF-A $_{165}$ & $21.15 \pm 2.69$ \\
& & \\
\hline
\end{tabular}

$\mathrm{IC}_{50}$ represents half maximal inhibitory concentration.

\section{Comparison of tumor growth inhibition by KP-VR2 and Aflibercept}

The inhibition of tumor growth by KP-VR2 and aflibercept was evaluated in a mouse xenograft model. Tumor cells were derived from diverse tissue origins (human LOVO colorectal adenocarcinoma cells, human SKUT-1B mesodermal tumor, and human HT-29 colorectal adenocarcinoma cells).

KP-VR2 significantly inhibited the growth of all three types of tumors (Fig. 5). To evaluate the antitumor effects of KP-VR2, we used the LOVO tumor model and treated them with either KP-VR2 or aflibercept at $1 \mathrm{mg} / \mathrm{kg}$. KP-VR2 treatment resulted in $84 \%$ and $88 \%$ reduction in tumor volume and weight, whereas aflibercept treatment caused a reduction of $66 \%$ and $73 \%$, respectively (Fig. 5A and 5B). We also assessed the antitumor effect of KP-VR2 on established SKUT-1B tumors. These results showed $80 \%$ and $51 \%$ reduction in tumor volume after $2 \mathrm{mg} / \mathrm{kg} \mathrm{KP-VR2}$ and aflibercept treatment, respectively (Fig. 5C and 5D), implying that KP-VR2 is a promising agent against tumors. In the study using HT-29 colorectal adenocarcinoma cells, a 3-fold lower dose KP-VR2 $(1 \mathrm{mg} / \mathrm{kg})$ was tested and found to be equally effective at inhibiting tumor growth and weight (Fig. 5E and 5F). Taken together, these results suggest that the improved avidity of KP-VR2 to VEGF-A and PIGF contributes to its higher efficacy in cancer treatment compared to aflibercept.

Figure 5A 


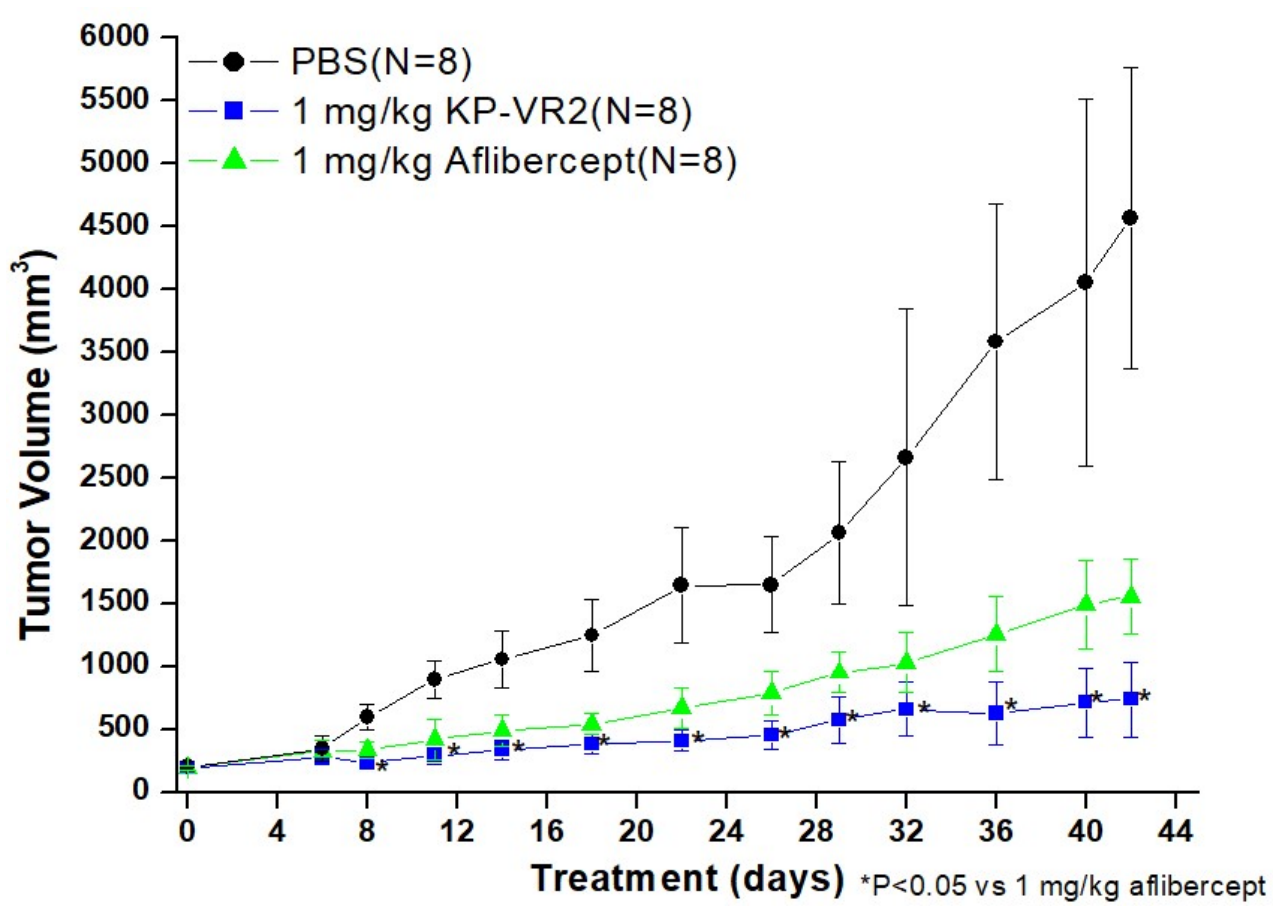

Figure 5B

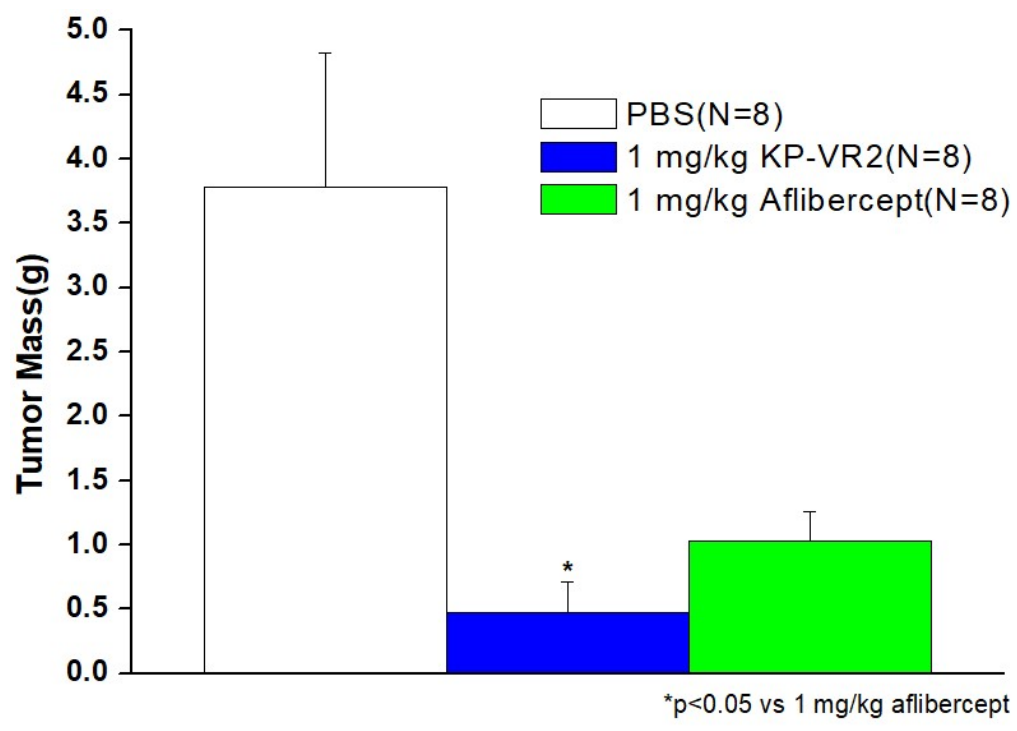

Figure 5C 


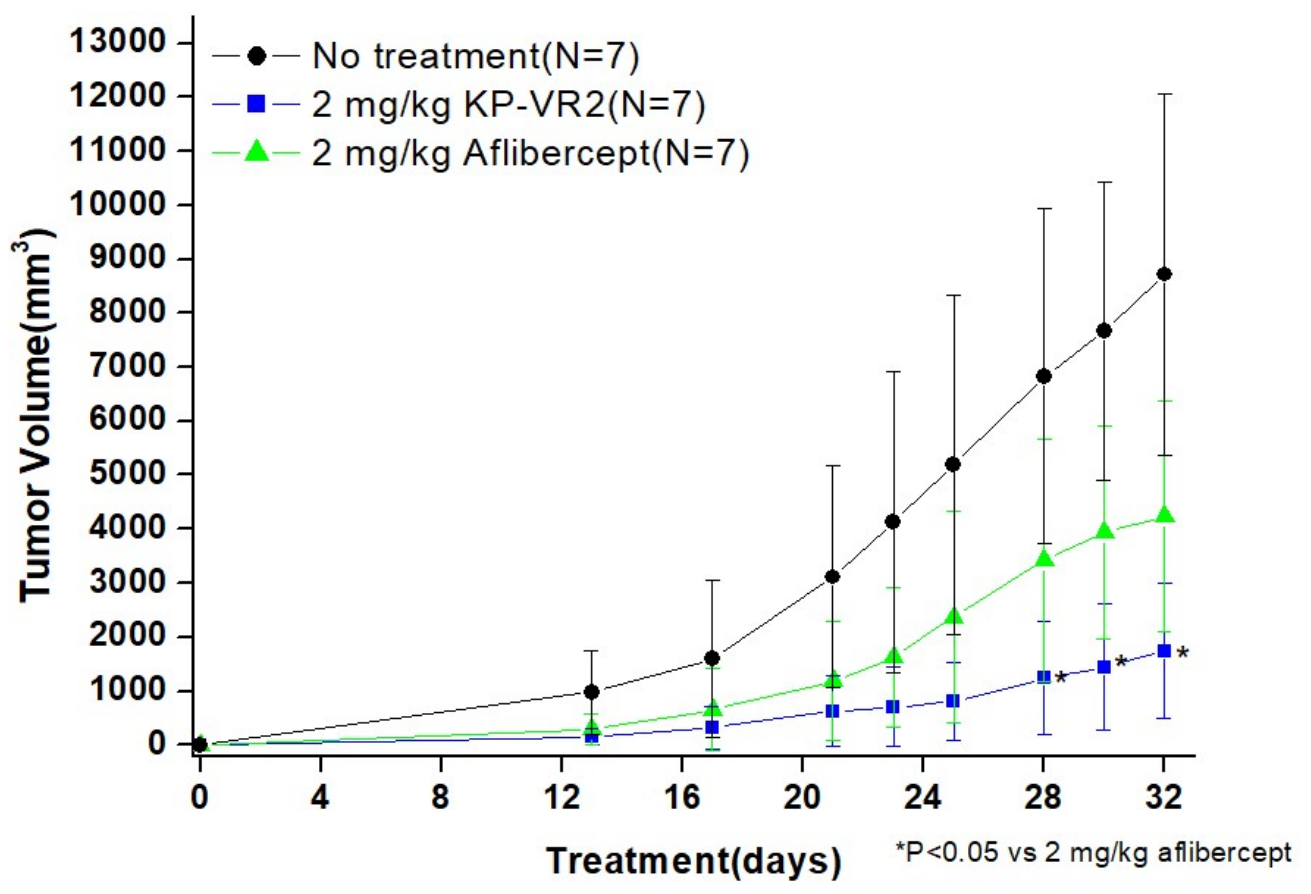

Figure 5D

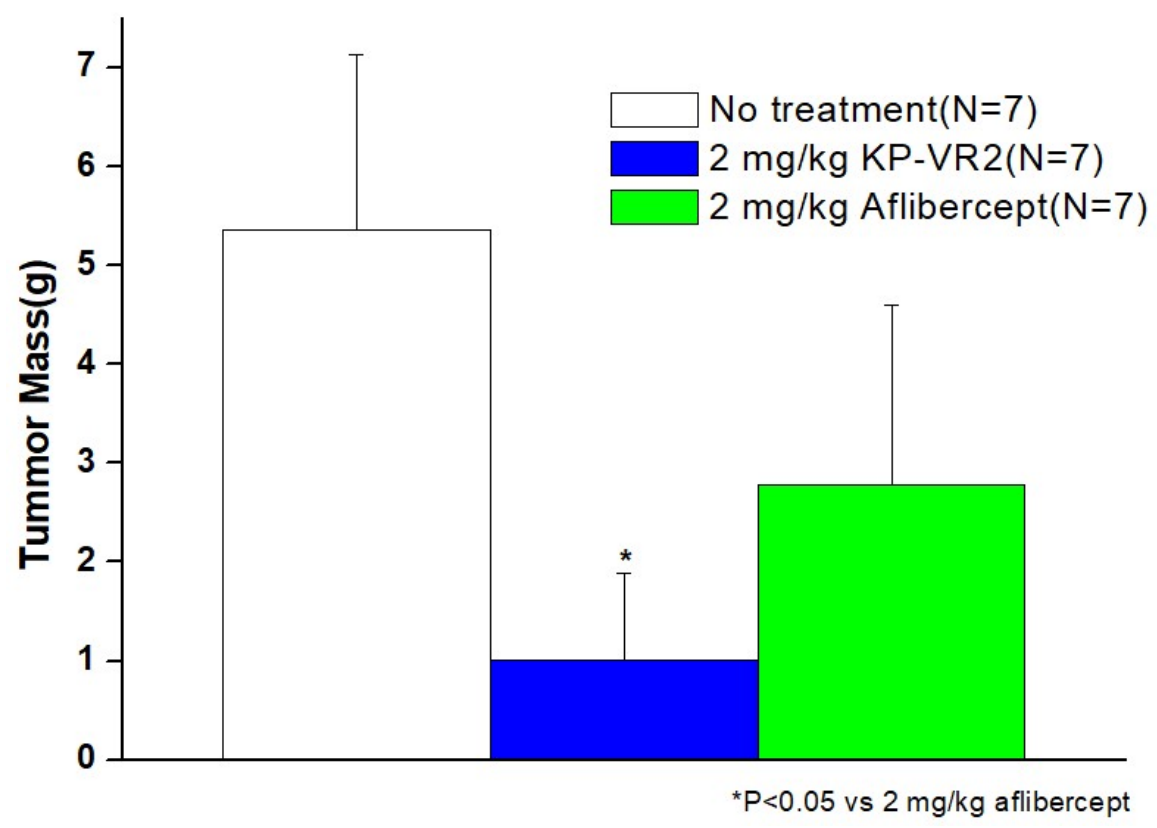


Figure 5E

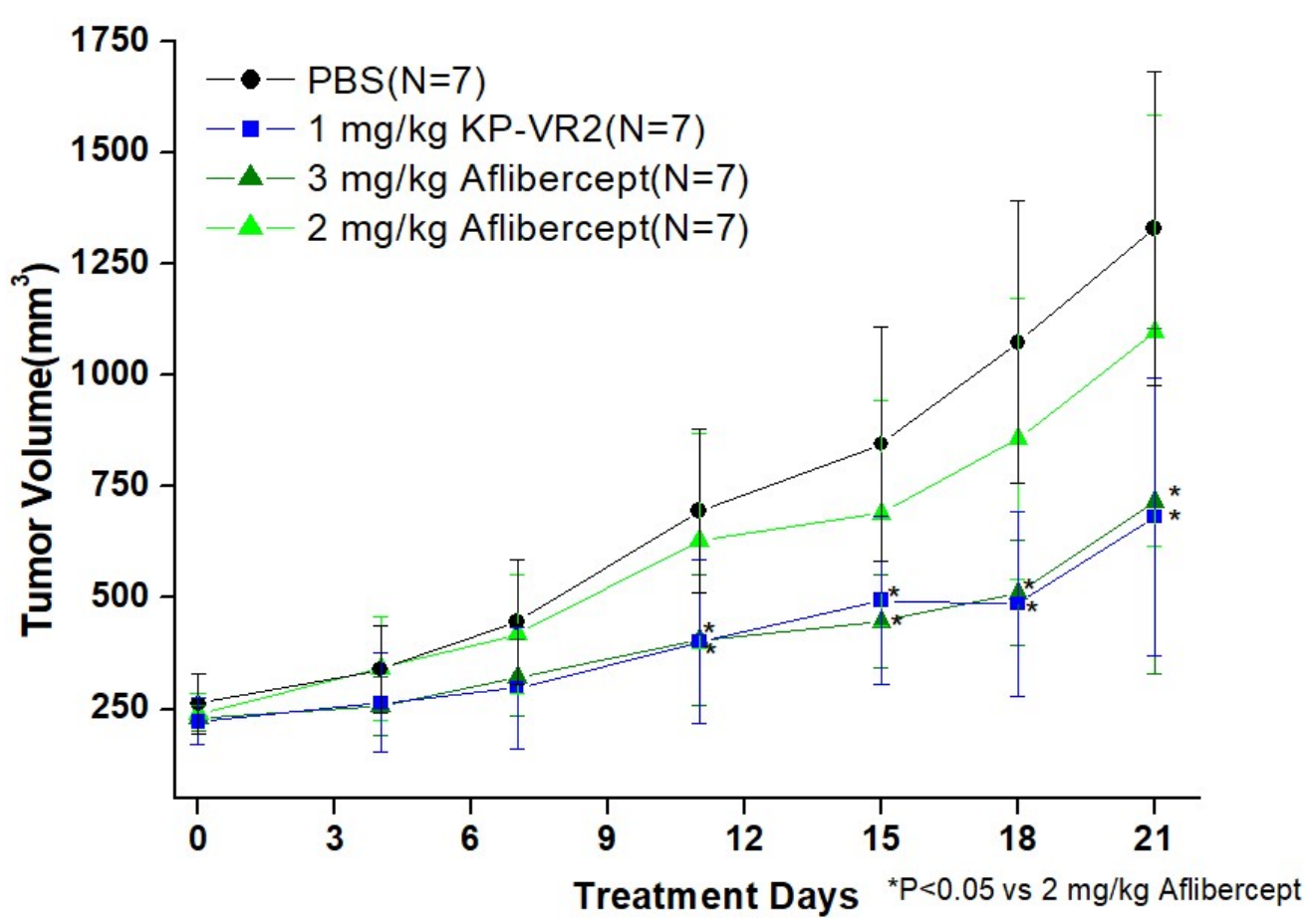

Figure 5F

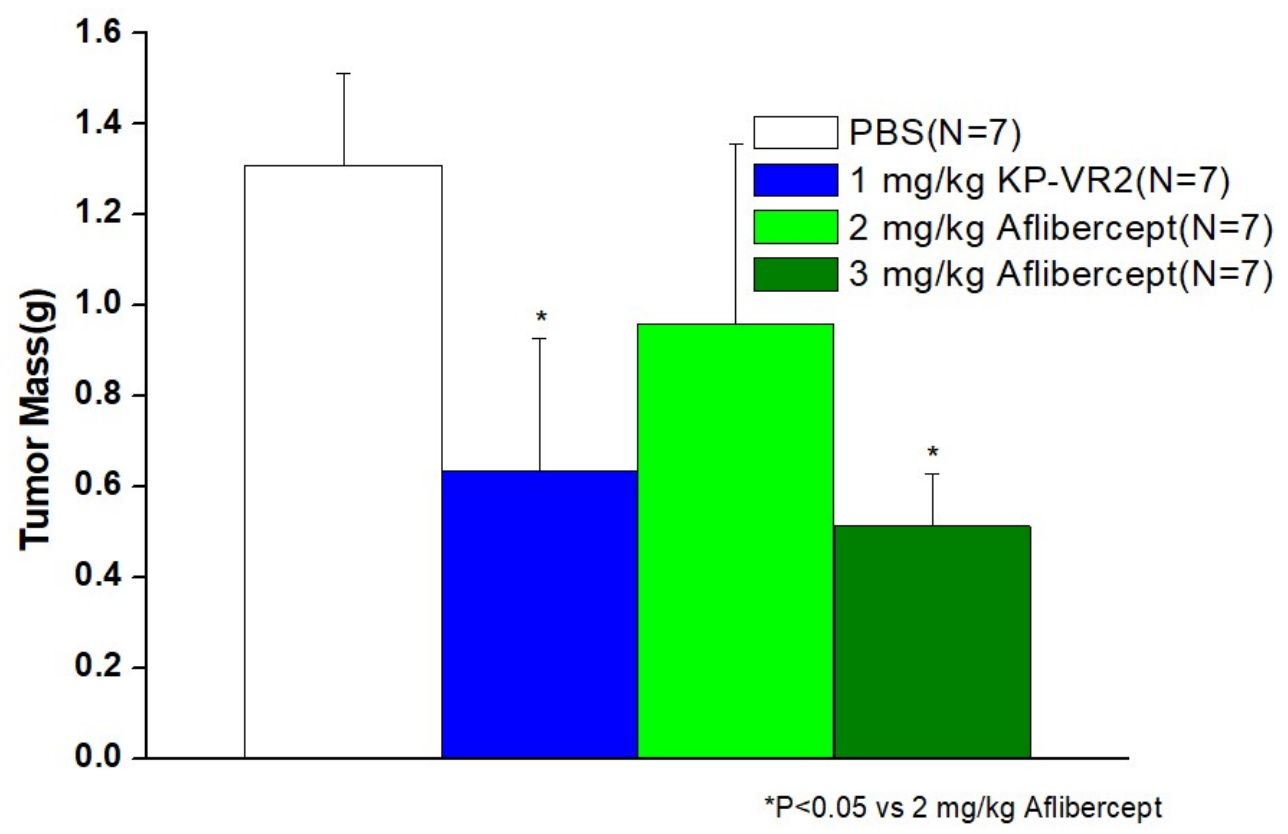


bioRxiv preprint doi: https://doi.org/10.1101/2021.02.02.429416; this version posted February 2, 2021. The copyright holder for this preprint (which was not certified by peer review) is the author/funder. All rights reserved. No reuse allowed without permission.

Figure 5. Inhibition of tumor growth in LOVO, SKUT-1B, and HT-29 tumors by KP-VR2 and aflibercept

Tumor growth inhibition induced by KP-VR2 in LOVO, SKUT-1B, and HT-29 models was compared with that of aflibercept. Tumor growth curves were plotted for KP-VR2, aflibercept, and control groups. Tumor volume (A) and mass (B) in LOVO tumor model. Tumor volume (C) and mass (D) in SKUT-1B tumor model. Tumor volume (E) and mass $(\mathrm{F})$ in HT-29 tumor model. * indicates $\mathrm{P}<0.05$, and all values are mean $\pm \mathrm{SD}$.

\section{Discussion}

Angiogenesis continues to be a viable therapeutic target for pathological conditions, including cancer and age-related macular degeneration. The development and investigation of bevacizumab and aflibercept as therapeutic agents has provided a basis for understanding the clinical potential of biological therapies that target angiogenesis.

The validation of VEGF as an important new target against cancer comes from pioneering clinical studies using a humanized monoclonal antibody that binds and blocks VEGF. Because anti-VEGF approaches act by blocking tumor-associated angiogenesis, which appears to be widely required by many different types of tumors, these approaches may prove to be generally useful against a wide assortment of cancers [22]. In addition, pathological angiogenesis seems to contribute to a number of non-neoplastic diseases, such as diabetic retinopathy [23] and psoriasis [24], extending the potential utility of anti-VEGF therapeutics. This highlights the need to optimize anti-VEGF approaches.

KP-VR2 (Fig. 1A) is an Fe fusion protein of two consecutive D2s from VEGFR-1, which was designed on the basis of a previously published report [25], in which the critical role of VEGFR-1 domain 2 was demonstrated to have high affinity binding.

VEGFR-1 has seven Ig-like domains in the extracellular domain (Fig. 1A). Among them, VEGFR-1 D2 is the primary contributor to VEGF-A and PlGF binding. Additionally, residues in VEGFR-1 D3 also participate in the high-affinity binding of VEGF-A and PIGF [26, 27]. Thus, the minimal required domain to bind both VEGF-A and PIGF with high affinity is VEGFR-1 D2-D3.

Although VEGFR-1 binds to VEGF-A and PIGF with higher affinity than VEGFR-2, the development of therapeutic decoy proteins with the VEGFR-1 backbone has proven to be difficult thus far. The major reason behind this is the high $\mathrm{pI}$ value of VEGFR-1 owing to the positively charged residues in the VEGFR-1 D3 region. This causes nonspecific extracellular matrix binding and poor pharmacokinetic profiles, leading to a shortened half-life, a subsequent decrease in efficacy, and even toxic side effects [25]. We assume that aflibercept employed VEGFR-2 D2 instead of VEGFR-1 D2 due to the intrinsic problem of VEGFR-1 D2.

However, previous structural analyses have indicated that VEGFR-1 might make greater use of its D2 in contacting VEGF [28]. Therefore, to rule out the problem of VEGFR-1 D3, we generated KP-VR2 consisting of two consecutive D2s of VEGFR-1, demonstrating improved decoy efficiency and dramatic increased avidity for both VEGF and PIGF.

Creating a new decoy receptor fusion protein with an additional binding site has great advantages. Most importantly, the two consecutive D2s of VEGFR-1 mainly consisted of two putative binding sites for ligands, resulting in greatly improved decoy efficiency, as shown in Fig. 2. The combination of highaffinity and improved avidity contributes to making KP-VR2 one of the most potent and efficacious VEGF blockers available. KP-VR2 showed more potent decoy activity against VEGF-A and PIGF than aflibercept. In addition, KP-VR2 showed more potent decoy activity against VEGF-A than 
bevacizumab, but bevacizumab was unable to bind PIGF. This was evidenced by our in vitro experiments demonstrating the strong suppression of proliferation and migration after KP-VR2 treatment. Consistent results were observed in vivo, where KP-VR2 showed much stronger antitumor effects in implanted tumor models when judged against aflibercept and bevacizumab.

When equimolar doses of KP-VR2 and aflibercept were compared in the LOVO, SKUT-1B, and HT29 xenograft models, it was apparent that much higher doses of aflibercept were required to inhibit tumor growth (Fig. 5). When used at the same dose, KP-VR2 showed better efficacy than aflibercept. Furthermore, KP-VR2 and aflibercept have similar circulation times in mice and rats (data not shown). Considering that the HT-29 tumor models are known to be relatively resistant to anti-VEGF therapy [27], these findings suggest the possibility of overcoming resistance to anti-VEGF therapy by concurrent blockade of PlGF with KP-VR2.

An additional advantage is that KP-VR2 is composed entirely of human sequences, minimizing the possibility of immunogenicity in human patients. Additionally, KP-R2 bound all species of VEGF tested, such as human, rabbit, rat, and mouse VEGF (data not shown), rendering it a versatile reagent that can be used in almost any experimental animal model.

Currently, anti-VEGF agents are approved for clinical use in combination with chemotherapy for the treatment of various tumors [22]. FDA approved aflibercept, in combination with leucovorin, 5fluorouracil, and irinotecan (FOLFIRI), for treating patients after progression with oxaliplatincontaining regimens $[2,16]$. This soluble decoy receptor shows one-to-one high-affinity binding to all isoforms of VEGF and PLGF [18, 29]. Aflibercept is also FDA approved for the treatment of agerelated macular degeneration (AMD), exhibiting higher efficiency than a single approach for the inhibition of VEGF-A [24].

In this study, KP-VR2 was designed to bind and sequester VEGF as well as PlGF with high affinity and avidity. Like aflibercept, KP-VR2 can be applied to treat various tumors and angiogenic ocular diseases, including AMD, and will be evaluated in future clinical trials. Our results suggest that KPVR2 is a potent and effective decoy for both VEGF and PlGF. Through the enhanced ligand binding avidity, KP-VR2 inhibited tumor growth and cell migration more effectively than did current VEGFTraps. The clinical applicability of this novel fusion protein should be further explored through preclinical and clinical studies.

\section{Experimental Procedures}

\section{Reagents and Materials}

Dulbecco's modified Eagle medium, fetal bovine serum (FBS), RPMI-1640, and trypsin-EDTA were purchased from Gibco (Gaithersburg, MD, USA). F12K and McCoy's 5A were purchased from ATCC (Rockville, MD, USA). Monoclonal antibodies against VEGFR-2, Erk1/2 phospho-VEGFR-2 (Tyr1175), and phospho-p44/42 MAPK (Erk1/2) (Thr202/Tyr204) were purchased from Cell Signaling Technology (Beverly, MA, USA). Anti-human IgG-peroxidase antibodies were purchased from KPL (Gaithersburg, MD, USA). Human VEGF-A ${ }_{165}$, human VEGF-A ${ }_{121}$, and human P1GF-1 were purchased from R\&D Systems (Minneapolis, MN, USA). Aflibercept (Regeneron Pharmaceuticals, Inc., Tarrytown, NY, USA) and bevacizumab (Genentech, Inc., San Francisco, CA, USA) were purchased.

\section{Cell Lines}

We used the following cells in the study: HUVECs (C-2519. Lonza, Walkersville, MD, USA), Chinese hamster ovary suspended cells (CHO-S; A1369601, Gibco, Gaithersburg, MD, USA), LOVO (CCL229, ATCC), SKUT-1B (HIB-115, ATCC), and HT-29 (HIB-38, ATCC). 
bioRxiv preprint doi: https://doi.org/10.1101/2021.02.02.429416; this version posted February 2, 2021. The copyright holder for this preprint (which was not certified by peer review) is the author/funder. All rights reserved. No reuse allowed without permission.

\section{Cell Culture}

HUVECs were cultured in EGM-2 medium supplemented with 2\% FBS, hydrocortisone, hFGFB, VEGF, R3-IGF, ascorbic acid, hEGF, GA-1000, and heparin. These cells were used to evaluate the effects of KP-VR2 on VEGFR-2.

LOVO, SKUT-1B, and HT-29 cells were grown in F12K, Eagle's Minimum Essential Medium, and McCoy's 5A or F12K with 10\% FBS, respectively. These cells were allowed to grow to a confluence of approximately $80 \%$ and were subcultured 2 or 3 times a week.

\section{Construction and Expression of KP-VR2}

KP-VR2 was created by fusing two consecutive second Ig domains of VEGFR-1 to Fc of human IgG1 (D2-D2-Fc). The KP-VR2 expression construct was transfected into CHO-S cells using Lipofectamine 2000 (Life Technologies) and cultured as previously reported [19]. KP-VR2 was secreted into the culture media and purified by protein A-Sepharose affinity chromatography, followed by two different cation and anion exchange chromatography runs.

The purified KP-VR2 was analyzed by non-reducing sodium dodecyl sulfate-polyacrylamide gel electrophoresis (SDS-PAGE) and western blotting. SDS-PAGE analysis of aflibercept and KP-VR2 was resolved on $4-12 \%$ gel under non-reducing conditions.

In the western blot analysis, the KP-VR2 and aflibercept were resolved on 4-12\% gel under nonreducing conditions, transferred to a polyvinylidene difluoride membrane, and the bands were probed with human Fc-specific antibody (KPL, Gaithersburg, MD, USA) and horseradish peroxidaseconjugated Goat anti-Human IgG (Seracare, Milford, MA, USA) and a DAB substrate kit (VECTOR Lab, Burlingame, CA, USA).

\section{Analysis of Binding of KP-VR2, Aflibercept, and Bevacizumab}

The binding abilities of KP-VR2, aflibercept, and bevacizumab were measured by ELISA. For this, a 96-well plate was coated with 1-3 nM of KP-VR2, aflibercept, and bevacizumab and blocked with $0.3 \%$ bovine serum albumin (BSA) in PBST (Phosphate-Buffered Saline Tween-20). Then, VEGF- $A_{165}$ and VEGF- $\mathrm{A}_{121}$ were added in gradually increasing amounts from $0.0625 \mathrm{nM}$ to $256 \mathrm{nM}$, and PLGF-1 from 0.1 to $2000 \mathrm{nM}$. Next, the plate was washed and reacted with the Goat anti-Human VEGF antibody. Then, the plate was washed again and reacted with peroxidase-conjugated anti-Goat Ig antibody. Next, 3,3',5,5'-tetramethylbenzidine (TMB) solution (Bethyl Laboratories, Montgomery, TX, USA) was added, and, thereafter, absorbance was measured at $450 \mathrm{~nm}$. The data were analyzed by a four-parameter logistic equation (Origin v7).

\section{Biolayer Interferometry (BLI) Analysis}

Binding kinetics were measured on the Octet QK384 System (ForteBio, Pall Life Science, Fremont, CA, USA), which is based on BLI technology.

KP-VR2 and aflibercept were immobilized onto anti-Human Fc biosensors (Pall ForteBio) at a concentration of $20 \mathrm{nM}$ for $600 \mathrm{~s}$, followed by biosensor rinsing in a kinetics buffer that served as a background buffer (KB buffer, Pall ForteBio). After they were balanced to the baseline, the biosensors were dipped into serial dilutions of VEGF and PlGF. The association was measured by the incubation of biosensors in various concentrations $(0.3125-20 \mathrm{nM})$ of VEGF and PIGF for $600 \mathrm{~s}$. Subsequently, dissociation was performed for $3,000 \mathrm{~s}$. The analyses of binding kinetics were evaluated by ForteBio data analysis software using curve fit models of 1:1 binding and 2:1 heterogeneous binding. 


\section{Cell Invasion Assay}

The HUVEC invasion assay was performed in vitro using a transwell chamber system with $8.0-\mu \mathrm{m}$ pore polycarbonate filter inserts (Corning Costar Corporation, Cambridge, MA, USA). Briefly, the $8-\mu \mathrm{m}$ pore inserts were coated with $5 \mu \mathrm{g} / \mathrm{mL}$ collagen (Collagen Type I, Rat Tail, Upstate). HUVECs $(7.5 \times$ $10^{4}$ cells $/ 300 \mu \mathrm{L}$ ) were seeded to the upper wells in EBM-2 medium with $0.1 \%$ FBS. EBM-2 medium $(750 \mu \mathrm{L})$, with $0.1 \%$ FBS in the presence of VEGF-A $165(350 \mathrm{ng} / \mathrm{mL})$ and KP-VR2, aflibercept, or bevacizumab (0-200 nM), was placed in the lower wells. The cells were incubated at $37{ }^{\circ} \mathrm{C}$ for $48 \mathrm{~h}$. After $48 \mathrm{~h}$, the upper collagen-coated surface was wiped off using a cotton swab. Cells that migrated through the filters were fixed, stained with crystal violet, photographed, and counted. The migrated cells were determined using a multi-detection microplate reader at an excitation of $458 \mathrm{~nm}$ and emission of $528 \mathrm{~nm}$ (BioTek Instrument, Inc., Winooski, VT, USA). The percentage of total migration (y-axis) was calculated as $\left(\mathrm{F}_{\text {Drug }}-\mathrm{F}_{\text {Basal }}\right) /\left(\mathrm{F}_{\text {Total }}-\mathrm{F}_{\text {Basal }}\right)$ where $\mathrm{F}_{\text {Total }}$ is fluorescence in the presence of VEGF$\mathrm{A}_{165}, \mathrm{~F}_{\text {Basal }}$ is fluorescence in the absence of VEGF- $\mathrm{A}_{165}$, and $\mathrm{F}_{\text {Drug }}$ is fluorescence in the presence of VEGF- $A_{165}$ mixed with drug at a specific molar ratio (x-axis) [20]. The values were analyzed by a fourparameter logistic equation (Origin v7).

\section{Tumor Growth Inhibition Experiments}

Tumor cells $\left(5.0 \times 10^{6}\right.$ cells/mouse) were suspended in PBS and implanted subcutaneously on the shaved right flank of $8-10$-week-old male BALB/c mice (Orient Bio Inc.). Tumor-bearing mice were randomized into control and treatment groups ( $n=7-8$ per group).

Then, the mice received an intraperitoneal injection of PBS, KP-VR2, or aflibercept twice weekly during the experiment. The size of the tumor was measured with calipers (tumor volume $=$ length $\times$ width $\times$ height).

For LOVO and HT-29 models, eight mice bearing tumors of 150-300 $\mathrm{mm}^{3}$ were injected intraperitoneally twice a week with $1 \mathrm{mg} / \mathrm{kg} \mathrm{KP}-\mathrm{VR} 2,1$ and $3 \mathrm{mg} / \mathrm{kg}$ aflibercept, and vehicle, $100 \mu \mathrm{L}$ of PBS. For the SKUT-1B model, the mice were allowed a brief recovery period (one day) and were then injected intraperitoneally twice a week with $2 \mathrm{mg} / \mathrm{kg} \mathrm{KP}-\mathrm{VR} 2$ and aflibercept until the end of the experiment, after which the animals were euthanized and tumors were excised and measured.

\section{Statistical Analyses}

Statistical differences between means were determined by an independent samples $t$ test. The statistical significance was set at $P<0.05$. 


\section{Data Availability Statement}

The data used to support the findings of this study are included within the article.

\section{Acknowledgements}

\section{Funding}

This work was supported by a grant from the Korea Health Technology R\&D Project through the Korea Health Industry Development Institute (KHIDI), funded by the Ministry of Health \& Welfare, Republic of Korea (Grant Number: HI18C1951). The funding organizations had no role in the design or conduct of this research. They provided unrestricted grants. The authors declare that they have no conflict of interest.

\section{Conflict of interest}

The authors declare that they have no conflicts of interest with the contents of this article. 
bioRxiv preprint doi: https://doi.org/10.1101/2021.02.02.429416; this version posted February 2, 2021. The copyright holder for this

\section{References}

1. Folkman, J., Merler, E., Abernathy, C., Williams, G. (1971) Isolation of a tumor factor responsible for angiogenesis. J. Exp. Med. 133, 275-288. 10.1084/jem.133.2.275

2. Giordano, G., Febbraro, A., Venditti, M., Campidoglio, S., Olivieri, N., Raieta, K., Parcesepe, P., Imbriani, G.C., Remo, A., Pancione, M. (2014) Targeting angiogenesis and tumor microenvironment in metastatic colorectal cancer: Role of aflibercept. Gastroenterol. Res. Pract. 10.1155/2014/526178

3. Saif, M.W. (2013) Anti-VEGF agents in metastatic colorectal cancer (mCRC): are they all alike? Cancer. Manag. Res. 5, 103-115. 10.2147/CMAR.S45193

4. Neufeld, G., Cohen, T., Gengrinovitch, S., Poltorak, Z. (1999) Vascular endothelial growth factor (VEGF) and its receptors. FASEB. J. 13, 9-22

5. Yancopoulos, G.D., Davis, S., Gale, N.W., Rudge, J.S., Wiegand, S.J., Holash, J. (2000) Vascular-specific growth factors and blood vessel formation. Nature. 407, 242-248. $10.1038 / 35025215$

6. Shibuya, M. (2010) Tyrosine kinase receptor Flt/VEGFR family: its characterization related to angiogenesis and cancer. Genes. Cancer. 1, 1119-1123.

$10.1177 / 1947601910392987$

7. Prager, G.W., Poettler, M., Unseld, M., Zielinski, C.C. (2012) Angiogenesis in cancer: anti-VEGF escape mechanisms. Transl. Lung. Cancer. Res. 1, 14-25. 10.3978/j.issn.22186751.2011.11.02

8. Claesson-Welsh, L., Welsh M (2013) VEGFA and tumour angiogenesis. J. Intern. Med. 273, 114-127. 10.1111/joim.12019

9. Joukov, V., Pajusola, K., Kaipainen, A., Chilov, D., Lahtinen, I., Kukk, E., Saksela, O., Kalkkinen, N., Alitalo, K. (1996) A novel vascular endothelial growth factor, VEGF-C, is a ligand for the Flt4 (VEGFR-3) and KDR (VEGFR-2) receptor tyrosine kinases. Embo. J. 15, 290-298

10. Jussila, L., Alitalo, K. (2002) Vascular growth factors and lymphangiogenesis. Physiol. Rev. 82, 673-700. 10.1152/physrev.00005.2002

11. McColl, B.K., Baldwin, M.E., Roufail, S., Freeman, C., Moritz, R.L., Simpson, R.J., Alitalo, K., Stacker, S.A., Achen, M.G. (2003) Plasmin activates the lymphangiogenic growth factors VEGF-C and VEGF-D. J. Exp. Med. 198, 863-868. 10.1084/jem.20030361

12. Khaliq, A., Li, X.F., Shams, M., Sisi, P., Acevedo, C.A., Whittle, M.J., Weich, H., Ahmed, A. (1996) Localisation of placenta growth factor (PIGF) in human term placenta. Growth. Factors. 13, 243-250. 10.3109/08977199609003225

13. Vuorela, P., Hatva, E., Lymboussaki, A., Kaipainen, A., Joukov, V., Persico, M.G., Alitalo, K., Halmesmaki, E. (1997) Expression of vascular endothelial growth factor and placenta growth factor in human placenta. Biol. Reprod. 56, 489-494.

10.1095/biolreprod56.2.489

14. Brave, S.R., Eberlein, C., Shibuya, M., Wedge, S.R., Barry, S.T. (2010) Placental growth factor neutralising antibodies give limited anti-angiogenic effects in an in vitro organotypic 
bioRxiv preprint doi: https://doi.org/10.1101/2021.02.02 429416; this version posted February 2, 2021. The copyright holder for this preprint (which was not certified by peer review) is the author/funder. All rights reserved. No reuse allowed without permission.

angiogenesis model. Angiogenesis. 13, 337-347. 10.1007/s10456-010-9190-0

15. Salem, M.E., El-Refai, S.M. (2013) Efficacy and safety of aflibercept and its role in the treatment of metastatic colorectal cancer. Rare. Cancers. Ther. 1, 3-19. 10.1007/s40487-013$0002-8$

16. Semeraro, F., Morescalchi, F., Duse, S., Parmeggiani, F., Gambicorti, E., Costagliola, C. (2013) Aflibercept in wet AMD: specific role and optimal use. Drug. Des. Devel. Ther. 7, 711-722. 10.2147/DDDT.S40215

17. Koh, Y.J., Kim, H.Z., Hwang, S.I., Lee, J.E., Oh, N., Jung, K., Kim, M., Kim, K.E., Kim, H., Lim, N.K., Jeon, C.J., Lee, G.M., Jeon, B.H., Nam, D.H., Sung, H.K., Nagy, A., Yoo, O.J., Koh, G.Y. (2010) Double antiangiogenic protein, DAAP, targeting VEGF-A and angiopoietins in tumor angiogenesis, metastasis, and vascular leakage. Cancer. Cell. 18, 171184. 10.1016/j.ccr.2010.07.001

18. Wiesmann, C., Fuh, G., Christinger, H.W., Eigenbrot, C., Wells, J.A., de Vos, A.M. (1997) Crystal structure at $1.7 \mathrm{~A}^{\circ}$ resolution of VEGF in complex with domain 2 of the Flt-1 receptor. Cell. 91, 695-704

19. Papadopoulos, N., Martin, J., Ruan, Q., Rafique, A., Rosconi, M.P., Shi, E., Pyles, E.A., Yancopoulos, G.D., Stahl, N., Wiegand, S.J. (2012) Binding and neutralization of vascular endothelial growth factor (VEGF) and related ligands by VEGF Trap, ranibizumab and bevacizumab. Angiogenesis. 15, 171-185. 10.1007/s10456-011-9249-6

20. Holash, J., Davis, S., Papadopoulos, N., Croll, S.D., Ho, L., Russell, M., Boland, P., Leidich, R., Hylton, D., Burova, E., Ioffe, E., Huang, T., Radziejewski, C., Bailey, K., Fandl, J.P., Daly, T., Wiegand, S.J., Yancopoulos, G.D., Rudge, J.S. (2002) VEGF-Trap: a VEGF blocker with potent antitumor effects. Proc. Natl. Acad. Sci. U.S.A., 99, 11393.

10.1073/pnas. 172398299

21. Anisimov, A., Leppanen, V.M., Tvorogov, D., Zarkada, G., Jeltsch, M., Holopainen, T., Kaijalainen, S., Alitalo, K. (2013) The basis for the distinct biological activities of vascular endothelial growth factor receptor-1 ligands. Sci. Signal. 6, ra52. 10.1126/scisignal.2003905

22. Lee, J.E., Kim, C., Yang, H., Park, I., Oh, N., Hua, S., Jeong, H., An, H.J., Kim, S.C., Lee, G.M., Koh, G.Y., Kim, H.M .(2015) Novel glycosylated VEGF decoy receptor fusion protein, VEGF-Grab, efficiently suppresses tumor angiogenesis and progression. Mol. Cancer. Ther. 14, 470. 10.1158/1535-7163.MCT-14-0968-T

23. Yoshida, A., Anand-Apte, B., Zetter, B.R. (1996) Differential endothelial migration and proliferation to basic fibroblast growth factor and vascular endothelial growth factor. Growth. Factors. 13, 57. 10.3109/08977199609034566

24. Mésange, P., Poindessous, V., Sabbah, M., Escargueil, A.E., de Gramont, A., Larsen, A.K. (2014) Intrinsic bevacizumab resistance is associated with prolonged activation of autocrine VEGF signaling and hypoxia tolerance in colorectal cancer cells and can be overcome by nintedanib, a small molecule angiokinase inhibitor. Oncotarget. 5, 4709-4721. 10.18632/oncotarget.1671

25. Ferrara, N., Hillan, K.J., Gerber, H.P., Novotny, W. (2004) Discovery and development of bevacizumab, an anti-VEGF antibody for treating cancer. Nat. Rev. Drug. Discov. 3, 391. $10.1038 / \operatorname{nrd} 1381$ 
26. Adamis, A.P., Miller, J.W., Bernal, M.T., D'Amico, D.J., Folkman, J., Yeo, T.K., Yeo, K.T. (1994) Increased vascular endothelial growth factor levels in the vitreous of eyes with proliferative diabetic retinopathy. Am. J. Ophthalmol. 118, 445-450. 10.1016/s00029394(14)75794-0

27. Anisimov, A., Leppänen, V.M., Tvorogov, D., Zarkada, G., Jeltsch, M., Holopainen, T., Kaijalainen, S., Alitalo, K. (2013) The basis for the distinct biological activities of vascular endothelial growth factor receptor-1 ligands. Sci. Signal. 6, ra52-ra52.

10.1126/scisignal.2003905

28. Detmar, M., Brown, L.F., Claffey, K.P., Yeo, K.T., Kocher, O., Jackman, R.W., Berse, B., Dvorak, H.F. (1994) Overexpression of vascular permeability factor/vascular endothelial growth factor and its receptors in psoriasis. J. Exp. Med. 180, 114. 10.1084/jem.180.3.1141

29. Stewart, M.W. (2012) Aflibercept (VEGF Trap-eye): the newest anti-VEGF drug. Br. J. Ophthalmol. 96, 1157. 10.1136/bjophthalmol-2011-300654 